\title{
Three-Dimensional Imaging Offers New Insights into Renal Pathology
}

\author{
H. H. Ward,* S. G. Clendenon, ${ }^{* *}$ J. M. Byars, ${ }^{* *}$ R.L. Bacallao, ${ }^{* * *}$ C. L. Phillips****
}

*Department of Pathology, University of New Mexico Health Sciences Center, MSC08-4640, 2325

Camino de Salud CRF 225, Albuquerque, NM 87131-5301

**Indiana Center for Biological Microscopy, Indiana University, 950 West Walnut, R2-202, Indianapolis, IN, 46202

***Departments of Medicine and Anatomy and Cell Biology, Indiana University, 950 West Walnut, R2-202, Indianapolis, IN, 46202

****Department of Pathology, Indiana University, Clarian Pathology Laboratory, 350 West 11th

Street, Room 4090, Indianapolis, IN 46202-4108

The kidneys of patients with renal disease display complex three-dimensional (3-D) alterations in renal architecture, and, in the case of inherited renal disease, abnormal renal development. Common histological approaches to pathological tissue evaluation include the use of standard light and epifluorescence microscopy combined with scanning and transmission electron microscopy. While traditional microscopy methods provide excellent 2-D datasets for evaluation, they do not permit detailed 3-D visualization of in-situ structures.

Mammalian kidney development involves the reciprocal induction of ureteric bud (UB) and metanephric mesenchyme resulting in formation of mature nephrons. We used anti-sense morpholino oligodeoxynucleotides (mODNs) to knockdown Tyro3 (a member of the Axl/Ufo receptor tyrosine kinase family) and Invs (a gene mutated in nephronophthisis type 2 patients [1]) in cultured mouse embryonic kidney rudiments to determine how these genes' products contribute to renal development. Rudiments were fluorescently labeled and imaged with a two-photon fluorescence microscope. The 3-D datasets were analyzed with segmentation and 3-D rendering software. We identified altered renal development in both the Invs (Table 1; Fig. 1) and Tyro3 mODN treated metanephric organ cultures. Rudiments treated with Invs mODNs showed altered arborization of the UB with wispy, back-arching ampullary tips and shortened distances between branch points. Tyro 3 knockdown resulted in a decrease in branching morphogenesis of UB segments in E12.5 kidneys but not in E11.5 kidneys. In both studies, rudiments cultured with control mODNs showed the expected pattern of UB branching with long limbs and blunt ampullary tips (Fig. 2). In our studies, the use of rudiment kidneys subjected to gene knockdown provided insights into the roles that Invs and Tyro3 play during kidney development.

In conclusion, confocal and multi-photon microscopy combined with 3-D rendering software provides pathologists and renal investigators with a 3-D picture of a large heterogeneous sample, thus allowing for improved interpretation and understanding of kidney development and renal disease processes.

References

[1] E.A. Otto et al., Nat Genet. 2003 Aug;34(4):413-20

[2] This work was supported by National Institutes of Health grants R21 DK067246, RO1 DK46833 and K08 DK02515. H.H. Ward is supported by a fellowship grant from the National Kidney Foundation. 
Table 1. Relative volume of branching UB (labeled with DBA-fluorescein) based on segmentation analysis performed using Amira 3.1 (http://www.amiravis.com, Visage Imaging) 3-D rendering software $(n=4$, concentration range of morpholino oligodeoxynucleotides $1.0 \mu \mathrm{M}-5.0 \mu \mathrm{M})$

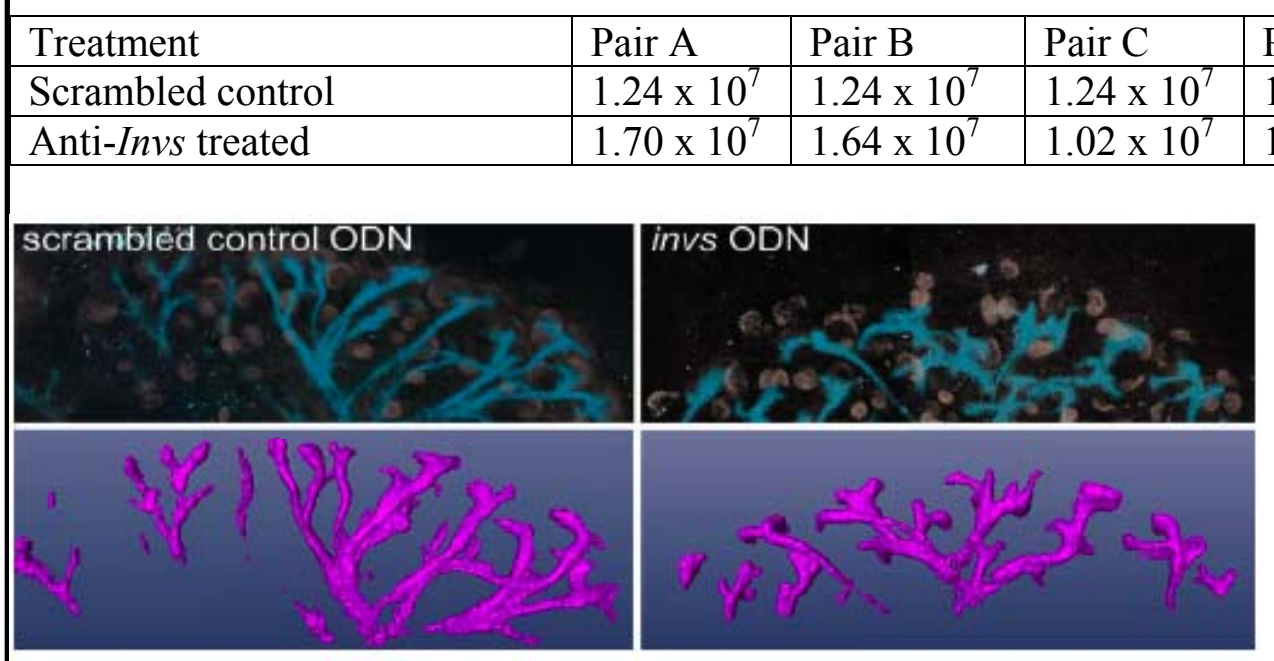

Fig. 1. Six days growth of anti-Invs and scrambled mODN-treated E12.5 kidneys. Compared to the control kidney, the kidney treated with anti-Invs mODN showed altered arborization of the UB (DBA lectin, blue in top panels) with wispy, back-arching ampullary tips and shortened distances between branch points. Glomeroid bodies were labeled with anti-Wilm's Tumor 1 (brown). (Top) Images rendered with Voxx. (Bottom) Amira segmentation of UB surface area labeled with Dilichos bifluoris lectin. Each image represents a stitched mosaic of three Z-series (20X water immersion objective).
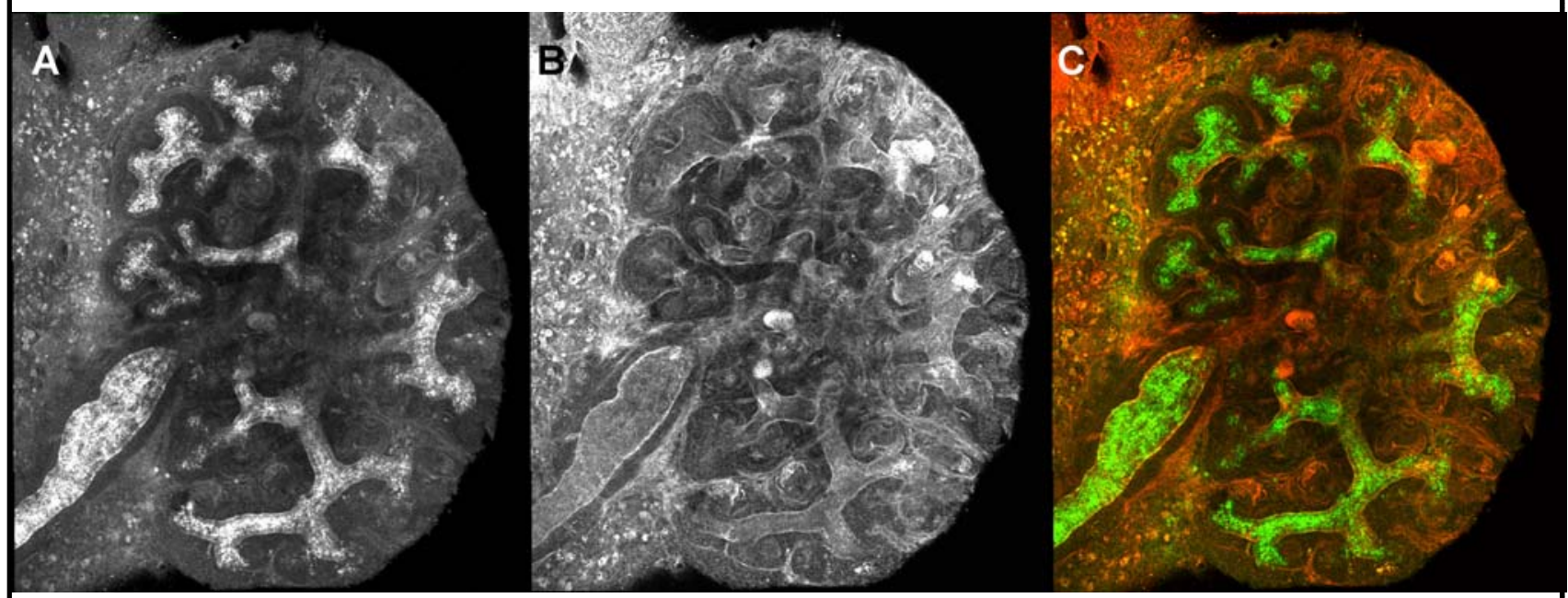

Fig. 2. UB and developing collecting ducts are labeled with (A) Dilichos bifluoris (DBA)-fluorescein and developing renal tubules with (B) peanut agglutinin (PNA)-rhodamine. (C) Merged composite of DBA (green) and PNA (red) labels. Z-stacks were collected on a two-photon fluorescence microscope (20X water immersion objective) and rendered with Voxx2 software (http://www.nephrology.iupui.edu/imaging/voxx, Indiana Center for Biological Microscopy) 